\title{
Action of Kanamycin Against Single and Dual Species Biofilms of Escherichia coli and Staphylococcus aureus
}

\author{
Ana Margarida Pereira, Ana Cristina Abreu, Manuel Simões*
}

LEPAE, Department of Chemical Engineering, Faculty of Engineering, University of Porto, 4200-465, Porto, Portugal

\begin{abstract}
Antibiotic resistance in one of the greatest threats to mankind, and prospects for the discovery of new antibiotic compounds are reduced. Due to their low-level of usage, aminoglycosides seem to have remained active against many pathogens and thus gained a renewed interest. In this work, we tested the effects of kanamycin against single and dual species biofilms of Escherichia coli and Staphylococcus aureus. The minimum inhibitory concentration (MIC), determined by the microdilution method, of $E$. coli and $S$. aureus were 4.5 and $3.5 \mathrm{mg} / \mathrm{L}$, respectively. The MIC was $5.0 \mathrm{mg} / \mathrm{L}$ for the dual species scenario. Biofilms were formed in 96-wells microtiter plates and their viability and biomass was assessed by alamar blue and crystal violet staining, respectively. Although the viability of sessile bacteria was greatly reduced with kanamycin (65\% and $67 \%$ for E. coli and S. aureus, respectively), their removal from surfaces was more difficult $(<35 \%)$. For dual species biofilms, the highest viability reduction obtained was $70 \%$ and the removal percentage was $15 \%$. Kanamycin was more efficient on biofilm killing than on their removal. Total biofilm killing and removal was not achieved even for kanamycin at $10 \times \mathrm{MIC}$ and resistance was more pronounced for the dual species biofilms. This highlights the greatest resistance capacity of biofilms when compared with planktonic cells. The overall results suggest that improved therapeutic strategies, including the application of higher doses or the combination with synergistic products, should be applied in biofilm control, particularly in polymicrobial biofilms.
\end{abstract}

Keywords Antibiotic Resistance, Biofilm Control, Kanamycin, Microdilution Method

\section{Introduction}

As the access to antibiotics became eased, they are being used to treat even the most common infection, usually in inadequate doses and time periods[1],[2]. The overuse and the misuse of these therapeutic agents readily led to the emergence and dissemination of antibiotic resistance bacteria, over the last several decades, which implies enormous health concerns, including the increased number of patients at risk, the increased severity of hospital- or community-acquired infections and also the failure of treatments.

The emergence of bacterial resistance is intensified when bacteria form biofilms. In the health context, some diseases and adverse medical conditions are now recognized to be the result of a biofilm infection (e.g. urinary tract infections, catheter infections and cystic fibrosis). In fact, over $80 \%$ of bacterial infections in humans involve the formation of biofilms[3]. Moreover, polymicrobial infections have been recurrent. In these type of infections, one microorganism generates a favourable niche for the infection and colonization of another one[4].

New antibiotics and strategies to prevent/overcome these

* Corresponding author:

mvs@fe.up.pt (Manuel Simões)

Published online at http://journal.sapub.org/microbiology

Copyright (C) 2012 Scientific \& Academic Publishing. All Rights Reserved uncontrolled outbreaks are urgently needed. However, very few new antibiotics were commercialized in the last decades. The discovery of the broad spectrum aminoglycoside family of antibiotics was a major breakthrough for medicine, but the emergence of resistant bacteria to these compounds and reports of their harmful side effects led to the decline of their use[5]. However, due to their low-level of usage in the last years, they appear to preserve their activity against diverse bacteria[6],[7]. Moreover, there has been a development of new strategies of dosage and molecular structure modifications to overcome their toxicity impasse[7]. The synergy of aminoglycosides with specific metabolites may also be an interesting strategy to eradicate persiter cells, commonly implicated in biofilms infections, and could contribute to the recycling of these older antibiotics, once considered ineffective[8]. In this study, the aminoglycoside antibiotic kanamycin was used on the control of single and dual biofilms of E. coli and S. aureus.

\section{Material and Methods}

\subsection{Bacteria and Minimum Inhibitory Concentration}

E. coli CECT 434 and S. aureus CECT 976 were used in this study. The MIC of single and dual species planktonic bacteria was determined by the microdilution method, according to McBain et al.[9]. 


\subsection{Biofilm Formation and Quantification of Biomass}

E. coli and $S$. aureus single and dual species biofilms were developed according to Simões et al.[10], with some modifications. Sterile 96-well flat tissue culture plates were filled with $200 \mu \mathrm{L}$ of cells at $1 \times 10^{8}$ cells $/ \mathrm{mL}$ in Mueller-Hinton broth (MHB, Merck, VWR, Portugal). The plates were incubated at $120 \mathrm{rpm}$ and $30{ }^{\circ} \mathrm{C}$ for $24 \mathrm{~h}$. Afterwards, the content of each well was carefully pipetted out and washed with $200 \mu \mathrm{L}$ of a sterile saline solution $(0.85 \% \mathrm{NaCl})$, to remove the non-adherent bacteria and to keep the osmotic pressure of biofilm cells. Biofilms were then exposed to 200 $\mu \mathrm{L}$ of different concentration of kanamycin (from 0.5 to 10 times the MIC), incubated at $120 \mathrm{rpm}$ and room temperature for $1 \mathrm{~h}$. Kanamycin solutions were refreshed every $20 \mathrm{~min}$, during the $1 \mathrm{~h}$ treatment. After treatment, kanamycin was discarded and cells were washed with $200 \mu \mathrm{L}$ of sterile water. Negative controls were done by incubation of the wells only with MHB, and positive controls were done by incubation of the wells with MHB and cells without antibiotic. Afterwards, the biofilms ( $24 \mathrm{~h}$ aged) were analysed in terms of biomass and viability.

The quantification of biofilm biomass was done using the crystal violet (Merck, VWR) assay as described by Presterl et al.[11]. Biofilm removal was given by:

$$
\% B . r .=\frac{O D_{C}-O D_{W}}{O D_{C}} x 100
$$

where $\%$ B.r. is the percentage of biofilm removal, $\mathrm{OD}_{\mathrm{C}}$ is the $\mathrm{OD}_{570 \mathrm{~nm}}$ value for the positive control and $\mathrm{OD}_{\mathrm{W}}$ is the $\mathrm{OD}_{570 \mathrm{~nm}}$ value for tested wells, assessed with a microplate reader (SpectraMax M2e from Molecular Devices).

\subsection{Biofilm Susceptibility}

To assess biofilm susceptibility, it was used a quick and very simple method employing the alamar blue dye and its property of being reduced by metabolically active cells from indigo blue to fluorescent pink[12],[13]. This method has actually been suggested by Peeters et al.[14] as one of the best substitutes of the colony forming units (CFU) counts. It was also suggested by several other authors since it is an easy, cost-effective, reproducible method used to assess sessile bacterial viability[15-17]. Recently Jiang et al. [18] used it in dual species biofilms. In this study, alamar blue assay was performed as described by Peeters et al.[19]. The fluorescence intensity was measured at an excitation wavelength of $570 \mathrm{~nm}\left(\lambda_{\text {exc }}=570 \mathrm{~nm}\right)$ and an emission wavelength of $590 \mathrm{~nm}$ $\left(\lambda_{\text {em }}=590 \mathrm{~nm}\right)$ with a microplate reader. The percentage of biofilm killing was given by:

$$
\% B . k .=\frac{F I_{C}-F I_{W}}{F I_{C}} \times 100
$$

where \%B.k. is the percentage of biofilm killing, $\mathrm{FI}_{\mathrm{C}}$ is the fluorescence intensity for the positive control and $\mathrm{FI}_{\mathrm{W}}$ is the fluorescence intensity value for tested wells.

To validate the results from the alamar blue assay the viability of biofilm cells was determined with the Live/Dead BacLight ${ }^{\mathrm{TM}}$ kit (Invitrogen) using epifluorescence microscopy (LEICA DMLB2 with a mercury lamp HBO/100W/3, incorporating a CCD camera to acquire images using IM50 software (LEICA) and a 100× oil immersion fluorescence objective), accordingly to Simões et al.[20]. Microscopic visualization also allowed the enumeration of bacteria in biofilm and the discrimination (due to their different shape, size and Gram coloration) of E. coli and $S$. aureus cells in dual species biofilms. The samples for microscopic preparation and the bacterial staining was performed according to Simões et al.[10].

\subsection{Statistical Analysis}

All experiments were done in triplicates. Because low samples numbers contributed to uneven variation, the results were analysed by the nonparametric Wilcoxon test. Results were presented as the means $\pm \mathrm{SD}$ (standard deviation) and $P$ $<0.05$ was considered statistically significant. Statistical analyses were performed with the statistical program SPSS 17.0 (Statistical Package for the Social Sciences).

\section{Results and Discussion}

Persistent infections are a major problem and a huge challenge, being responsible for significant numbers of deaths. Antimicrobial resistance events are amplified when bacteria form biofilms. The main strategies to reduce biofilm-associated infections include: prevention of initial contamination; minimization of microbial cell attachment, use of antimicrobials to penetrate the biofilm matrix and inactivate the embedded microorganisms or, at worst, removal of the infected device[3]. Removal of infected devices is unavoidable when the antimicrobial strategy fails on biofilm control. Accordingly, new chemotherapeutic strategies are required in order to circumvent the antimicrobial resistance issue. One reason for the limited clinical utility of antibiotic susceptibility tests may be that bacteria exist as a biofilm in many infections but, in these methods, are grown planktonically in vitro. Treatment of biofilm-associated infections with the existing approved therapies remains a significant medical challenge[21]. In fact, antimicrobial chemotherapeutic tests with biofilms are scarce[3].

Due to their low-level of usage over the years, aminoglycoside antibiotics seem to have preserved their activity against many resistant bacteria, which gives them a renewed interest[6]. In this work, the susceptibility of single and dual species biofilms of $E$. coli and $S$. aureus was tested. Although the efficacy of kanamycin against $E$. coli biofilms has already been studied[22], to our knowledge there are no studies on the efficacy of kanamycin to control E. coli and $S$. aureus single and dual species biofilms.

\subsection{MIC against planktonic $E$. coli and $S$. aureus}

The MIC of kanamycin against $E$. coli and $S$. aureus was 4.5 and $3.5 \mathrm{mg} / \mathrm{L}$, respectively (data not shown). These values show that kanamycin has higher activity against $S$. aureus than against $E$. coli $(P<0.05)$. Aminoglycoside antibiotics mode of action consists in the inhibition of pro- 
tein synthesis and disruption of the cytoplasmic membrane[23]. Bacterial resistance to aminoglycosides can occur by modification of the molecular target, synthesis of aminoglycoside-modifying enzymes and also by modification of aminoglycoside transport (import and efflux)[23]. Gram-negative organisms are the only ones to have the resistance nodulation division (RND) family of efflux systems, which is the predominant mechanism associated with antibiotic resistance[23].

The MIC for a dual culture of E. coli and S. aureus was 5.0 $\mathrm{mg} / \mathrm{L}$. While MIC values for single E. coli and dual cultures are not statistically significant $(P>0.05)$, the MIC for $S$. aureus is statistically different from the value obtained for the dual species culture $(P<0.05)$.

\subsection{Biofilm Formation and Susceptibility}

Analysis of biofilms formed in the microtiter plates showed that $E$. coli and $S$. aureus biofilms had $1.2 \times 10^{8}$ cells and $2.7 \times 10^{8}$ cells per well, respectively. Dual species biofilms had a density of $3.1 \times 10^{8}$ cells per well. The assessment of the total number of cells in single and dual species biofilms was performed by Live/Dead staining as previously explained. This staining also demonstrated that dual species biofilms were only composed by $E$. coli.

The susceptibility of biofilms to kanamycin was tested for multiple values of the MIC and the biofilm killing and removal percentages obtained are demonstrated in Figures 1 and 2 , respectively. There is a general reduction of viability of $E$. coli with the increase of kanamycin concentration $(P<$ $0.05)$, which reaches a maximum $(65 \%$ reduction) at $7 \times$ MIC (Fig. 1) $(P<0.05)$. A higher concentration of kanamycin does not seem to further reduce the viability of biofilms $(P>0.05)$. S. aureus biofilms show the same profile of reduction of kanamycin susceptibility $(P<0.05)$, but the maximum value of viability reduction $(67 \%$ reduction $)$ was achieved at $10 \times$ MIC $(P<0.05)$. For the dual species biofilms, the maximum viability reduction $(70 \%$ reduction) was achieved for a concentration of $10 \times \operatorname{MIC}(P<0.05)$. The alamar blue assay results are in agreement $(P>0.05)$ with those obtained by Live/Dead staining (data not shown).

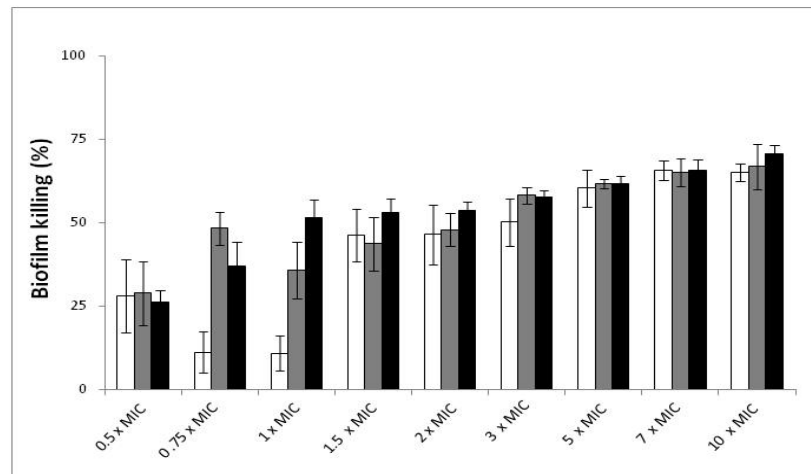

Figure 1. Killing (\%) of E. coli $(\square)$ and S. aureus ( $\square$ ) single and dual species ( $\square$ ) biofilms after treatment with kanamycin at various MIC multiple concentrations. MIC for $E$. coli $=4.5 \mathrm{mg} / \mathrm{L}$; MIC for $S$. aureus $=$ $3.5 \mathrm{mg} / \mathrm{L}$; MIC for $E$. coli and $S$. aureus co-culture $=5.0 \mathrm{mg} / \mathrm{L}$. Mean values $\pm \mathrm{SD}$ for at least three replicates are illustrated
Regarding the biomass reduction (Fig. 2), the increase of kanamycin concentration does not increase biofilm mass reduction for $E$. coli and $S$. aureus single and dual species biofilms $(P>0.05)$. Biofilm mass reduction was higher for $S$. aureus biofilms (with the maximum of $35 \%$ of biofilm mass reduction) and lower for dual species biofilms (with the ma ximum of $15 \%$ of biofilm mass reduction). The percentage of biofilm removal was significantly lower $(P<0.05)$ for the dual species biofilms.

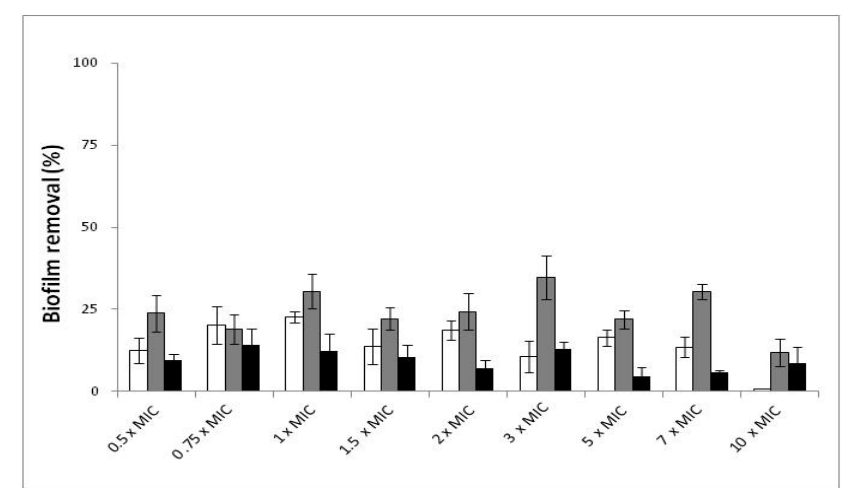

Figure 2. Biomass removal (\%) of E. coli ( $\square$ ) and S. aureus ( $\square$ ) single and dual species ( $\square$ ) biofilms after treatment with kanamycin at various MIC multiple concentrations. MIC for $E$. coli $=4.5 \mathrm{mg} / \mathrm{L}$; MIC for $S$. aureus $=3.5 \mathrm{mg} / \mathrm{L}$; MIC for $E$. coli and $S$. aureus co-culture $=5.0 \mathrm{mg} / \mathrm{L}$. Mean values $\pm \mathrm{SD}$ for at least three replicates are illustrated

Kanamycin was applied at concentrations from sub- inhibitory to $10 \times$ MIC against biofilms. A concentration of 10 $\times$ MIC was not effective to control (kill and remove) the single and the dual species biofilms. These results highlight the fact that MIC values cannot be taken as reference in biofilm infections and that cells in biofilm can be much more resistant. In addition to the resistance mechanisms found in planktonic cells (such as efflux pumps, cellular impermeability imparted by the outer layers, enzymes that confer resistance and natural evolutionary mutations) there are six interesting hypothesized mechanisms that help to explain the increased resistance of biofilms to antimicrobials: the direct interaction between the biofilm extracellular polymeric substances and antimicrobials, affecting diffusion and availability; the existence of an altered chemical microenvironment within the biofilm leading to areas of reduced or no growth (dormant cells); the development of biofilmspecific phenotypes; the ability of microorganisms in biofilms to express specific resistance genes; the possibility of damaged bacterial cells undergoing programmed cell death; the existence of persister cells. ${ }^{3}$

This study also demonstrates that biofilm killing and removal are distinct phenomena. Even if it is possible to reduce the viability of sessile bacteria $(>65 \%)$, their removal from surfaces is extremely difficult $(<35 \%)$. This difficulty in biofilm removal with diverse antimicrobial products was already reported by Chen and Stewart[24]. These authors found that some treatments caused significant killing but not much removal while other antimicrobial treatments caused removal with residual killing[24]. Furthermore, resistance to removal seems to increase with species association. In this 
study, the association of E. coli and $S$. aureus in biofilm formation induced the formation of biofilms with only $E$. coli cells and with a higher cell density, compared with the single species scenario. Burmølle et al.[25] already suggested that, even in polymicrobial infections, bacteria grow in monospecies biofilms. Different bacterial species may form sovereign aggregates in the same infection, but they do it in different locations of the infection and the sovereign bacterial aggregate is mainly composed by one species. The higher cell density obtained can account for the increase resistance to removal. Furthermore, the putative transfer of resistance genes from $S$. aureus to $E$. coli can contribute for the higher resistance of the biofilms formed by the initial dual species association. The gene transfer between species in biofilms has already been reported by Weigel et al.[26]. There are evidences that species association can increase biofilm resistance and resilience to antimicrobial exposure[10],[20] and it seems important to take this aspect into account when developing therapeutic strategies.

\section{Conclusions}

The results show that kanamycin at concentrations from sub-inhibitory to $10 \times$ MIC was not efficient for the control of $E$. coli and $S$. aureus biofilm cells. It is common that, in the presence of therapeutically available antibiotic concentrations, biofilms remain viable after treatment. In fact, antimicrobial concentrations necessary to inhibit bacterial biofilms can be up to 10-1000 times higher than those needed to inhibit the same bacteria grown planktonically[27]. Biofilms initially formed by both species were more resistant to removal after treatment with kanamycin than their single species counterparts. Also, the antibiotic was more efficient on biofilm killing than on their removal. Biofilm resistance is usually multifactorial and may vary from one organism to another. This combination of factors makes biofilm eradication difficult with a single target antimicrobial. It is possible that the synergistic application of kanamycin with other antibiotics acting on distinct bacterial targets can have a wider role in biofilm control.

\section{ACKNOWLEDGEMENTS}

The authors would like to acknowledge the Operational Programme for Competitiveness Factors - COMPETE and FCT - the Portuguese Foundation for Science and Technology through Project Bioresist - PTDC/EBB-EBI/105085 $/ 2008$, for supporting this work.

\section{REFERENCES}

[1] Alanis, A.J. 2005. Resistance to antibiotics: Are we in the post-antibiotic era? Arch. Med. Res. 36:697-705
[2] Levy, S.B. 2001. Antibiotic resistance: Consequences of inaction. Clin. Infect. Dis. 33:S124-S129.

[3] Simões, M. 2011. Antimicrobial strategies effective against infectious bacterial biofilms. Curr. Med. Chem. 18:2129-2145.

[4] Brogden, K.A., J.M. Guthmiller, and C.E. Taylor. 2005. Human polymicrobial infections. Lancet. 365:253-255.

[5] Hermann, T. 2007. Aminoglycoside antibiotics: old drugs and new therapeutic approaches. Cell. Mol. Life Sci. 64:1841-1852

[6] Gad G.F., H.A. Mohamed, and H.M. Ashour. 2011. Aminoglycoside resistance rates, phenotypes, and mechanisms of gram-negative bacteria from infected patients in upper Egypt. PLoS ONE. 6(2):e17224.

[7] Falagas, M.E., A.P. Grammatikos, and A. Michalopoulos. 2008. Potential of old-generation antibiotics to address current need for new antibiotics. Expert. Rev. Anti. Infecti. Ther. 6:593-600.

[8] Allison, K.R., M.P. Brynildsen, and J.J. Collins. 2011. Metabolite-enabled eradication of bacterial persisters by aminoglycosides. Nature. 473:216-220.

[9] McBain, A.J., R. G. Ledder, P. Sreenivasan, and P. Gilbert. 2004. Selection for high-level resistance by chronic triclosan exposure is not universal. J. Antimicrob. Chemother. 53:772-777.

[10] Simões, L.C., M. Simões, and M.J. Vieira. 2010. Influence of the diversity of bacterial isolates from drinking water on resistance of biofilms to disinfection. Appl. Environ. Microbiol. 76:6673-6679.

[11] Presterl, E., A. J. Grisold, S. Reichmann, A. M. Hirschl, A. Georgopoulos and W. Graninger. 2005. Viridansstreptococci in endocarditis and neutropenic sepsis: biofilm formation and effects of antibiotics. J. Antimicrob. Chemother. 55:45-50.

[12] O'Brien, J., I. Wilson, T. Orton, and F. Pognan. 2000. Investigation of the alamar blue (resazurin) fluorescent dye for the assessment of mammalian cell cytotoxicity. Eur. J. Biochem. 267:5421-5426.

[13] Palomino, J.C., A. Martin, M. Camacho, H. Guerra, J. Swings, and F. Portaels. 2002. Resazurin microtiter assay plate: simple and inexpensive method for detection of drug resistance in Mycobacterium tuberculosis. Antimicrob. Agents. Chemother. 46:2720-2722.

[14] Peeters, E., H.J. Nelis, and T. Coenye. 2008. Comparison of multiple methods for quantification of microbial biofilms grown in microtiter plates. J. Microbiol. Methods. 72:157-165.

[15] Mariscal, A., R.M. Lopez-Gigosos, \& M. Carnero-Varo, and J. Fernandez-Crehuet. 2009. Fluorescent assay based on resazurin for detection of activity of disinfectants against bacterial biofilm. Appl. Microbiol. Biotechnol. 82: 73-783.

[16] Peeters, E., H.J. Nelis, and T. Coenye. 2008. Evaluation of the efficacy of disinfection procedures against Burkholderia cenocepacia biofilms. J. Hosp, Infect. 70:361-368.

[17] Pettit, R.K., C.A. Weber, M.J. Kean, H. Hoffmann, G.R. Pettit, R. Tan, K.S. Franks, and M.L. Horton. 2005. Microplate alamar blue assay for Staphylococcus epidermidis 
biofilm susceptibility testing. Antimicrob. Agents Chemother. 49:2612-2617.

[18] Jiang, L.M., M.A. Hoogenkamp, L.W.M. van der Sluis, P.R. Wesselink, W. Crielaard, and D.M. Deng. 2011. Resazurin metabolism assay for root canal disinfectant evaluation on dual-species biofilms. JOE. 37:31-35.

[19] Peeters, E., H.J. Nelis, and T. Coenye. 2008. Resistance of planktonic and biofilm-grown Burkholderia cepacia complex isolates to the transition metal gallium. J. Antimicrob. Chemother. 61:1062-1065.

[20] Simões, M., L.C. Simões, and M.J. Vieira. 2009. Species association increases biofilm resistance to chemical and mechanical treatments. Water Res. 43:229-237.

[21] Lynch AS, and D. Abbanat. 2010. New antibiotic agents and approaches to treat biofilm-associated infections. Expert. Opin. Ther. Pat. 20:1373-1387.

[22] Ito, A., A. Taniuchi, T.M., K. Kawata, and S. Okabe. 2009. Increased antibiotic resistance of Escherichia coli in mature biofilms. Appl. Environ. Microbiol. 75:4093-4100.

[23] Arya, D.P. (2007). Aminoglycoside antibiotics: from chemical biology to drug discovery. 1st ed. John Wiley \& Sons, Inc, New Jersey.

[24] Chen, X., and P.S. Stewart. 2000. Biofilm removal caused by chemical treatments. Water Res. 34:4229-4233.

[25] Burmølle, M., T.R. Thomsen, M. Fazli, I. Dige, L. Christensen, P. Homøe, M. Tvede, B. Nyvad, T. Tolker-Nielsen, M. Givskov, C. Moser, K. Kirketerp-Møller, H.K. Johansen, N. Høiby, P.Ø. Jensen, S.J. Sørensen, and T. Bjarnsholt. 2010. Biofilms in chronic infections - a matter of opportunity monospecies biofilms in multispecies infections. FEMS Immunol. Med. Microbiol. 59:324-336.

[26] Weigel, L.M., R.M. Donlan, D.H. Shin, B. Jensen, N.C. Clark, L.K. McDougal, W. Zhu, K.A. Musser, J. Thompson, D. Kohlerschmidt, N. Dumas, R.J. Limberger, and J.B. Patel. 2007. High-level vancomycin-resistant Staphylococcus aureus isolates associated with a polymicrobial biofilm. Antimicrob. Agents. Chemother. 51:231-238.

[27] Gilbert, P., and D.G. Allison, McBain AJ. 2002. Biofilms in vitro and in vivo: Do singular mechanisms imply crossresistance? J. Appl. Microbiol. Symp. Suppl. 292:98S-110S. 